\title{
Calcium Gluconate-derived Ultrathin Carbon Nanosheets for High-Efficient Capacitive Energy Storage
}

\author{
Yamin Zhang ${ }^{\text {b, c }}$, Zhongpu Wang ${ }^{\text {b }}$, Deping Li ${ }^{\text {a, b * }}$, Qing Sun ${ }^{\text {b }}$, Kangrong Lai ${ }^{\text {c }}$, Kaikai Li a , \\ Qunhui Yuan ${ }^{\mathrm{a}}$, Xingjun Liu ${ }^{\mathrm{a}}$ and Lijie $\mathrm{Ci}$ a, b*
}

\begin{abstract}
a State Key Laboratory of Advanced Welding and Joining, School of Materials Science and Engineering, Harbin Institute of Technology, Shenzhen, 518055, China

${ }^{\mathrm{b}}$ Research Center for Carbon Nanomaterials, Key Laboratory for Liquid-Solid Structural Evolution \& Processing of Materials (Ministry of Education), School of Materials Science and Engineering, Shandong University, Jinan, 250061, China

c Department of Physics, Changji University, Changji 831100, China
\end{abstract}

Address correspondence to cilijie@hit.edu.cn; lideping@hit.edu.cn 


\begin{abstract}
Porous carbon has attracted extensive attentions as the electrode material for various energy storage devices considering its advantages like high theoretical capacitance/capacity, high conductivity, low cost and earth abundant inherence. However, there still exists some disadvantages limiting its further applications, such as the tedious fabrication process, limited metal-ion transport kinetics and undesired structure deformation at harsh electrochemical conditions. Herein, we report a facile strategy, with calcium gluconate firstly reported as the carbon source, to fabricate ultrathin porous carbon nanosheets. The as-prepared Ca-900 electrode delivers excellent K-ion storage performance including high reversible capacity $\left(497.7 \mathrm{mAh} \mathrm{g}^{-1}\right)$, superior rate capability (154.8 $\mathrm{mAh} \mathrm{g}^{-1}$ at an ultrahigh current density of $5.0 \mathrm{~A} \mathrm{~g}^{-1}$ ) and ultra-stable long-term cycling stability (a high capacity retention ratio of $\sim 72.4 \%$ after 4000 cycles at $1.0 \mathrm{~A} \mathrm{~g}^{-1}$ ). Similarly, when being applied in $\mathrm{Zn}$-ion capacitors, the Ca-900 electrode also exhibits an ultra-stable cycling performance with $\sim 90.9 \%$ capacity retention after 4000 cycles at $1.0 \mathrm{~A} \mathrm{~g}^{-1}$, illuminating the applicable potentials. Moreover, the origin of the fast and smooth metal-ion storage is also revealed by carefully designed consecutive $\mathrm{CV}$ measurements. Overall, considering the facile preparation strategy, unique structure, application flexibility and in-depth mechanism investigations, this work will deepen the fundamental understandings and boost the commercialization of high-efficient energy storage devices like potassium-ion/sodium-ion batteries, zinc-ion batteries/capacitors and aluminum-ion batteries.
\end{abstract}

Key words: In situ self-template fabrication strategy, Ultrathin porous carbon nanosheets, Potassium-ion batteries, Zinc-ion hybrid capacitors, Capacitive energy storage 


\section{Introduction}

Lithium-ion batteries (LIBs), as the representative secondary battery systems, have been triumphantly used in electric vehicles, portable electronics, and smart grids ${ }^{1,2}$. However, with the development of technologies, the dependence on LIBs has led to the rising cost and reducing availability of lithium resources in the crust $^{3}$. Therefore, it is urgent to find alternatives to LIBs. Sodium-ion batteries (SIBs) have become the focus of attention because of their earth-abundant inherence and similar electrochemical properties to $\mathrm{LIBs}^{4,5}$. Nevertheless, sodium ions are difficult to be reversibly inserted into the graphite anode, limiting its commercialization prospect.

Potassium-ion batteries (PIBs), rooting in competitive crust reserves $(\sim 15000 \mathrm{ppm})$ and feasible K-ion insertion/extraction in commercial graphite electrode, are attracting enormous attentions. Besides, the redox potential of $\mathrm{K}^{+} / \mathrm{K}(-2.93 \mathrm{~V})$ is lower than that of $\mathrm{Na}^{+} / \mathrm{Na}(-2.71 \mathrm{~V})$, and close to $\mathrm{Li}^{+} / \mathrm{Li}(-3.04 \mathrm{~V})$, indicating that PIBs can work within a high voltage platform and deliver a high energy density ${ }^{6,7}$. In addition, in organic solvent electrolytes, such as propylene carbonate (PC), $\mathrm{K}^{+} / \mathrm{K}(-2.88 \mathrm{~V})$ exhibits the lowest redox potential compared to $\mathrm{Li}^{+} / \mathrm{Li}(-2.79 \mathrm{~V})$ and $\mathrm{Na}^{+} / \mathrm{Na}$ $(-2.56 \mathrm{~V})^{8,9}$. Despite a larger ionic radius of K-ion, owing the weak Lewis acidity of K-ion, the radius of solvated $\mathrm{K}$-ion is the smallest as compared to $\mathrm{Li}$-ion and $\mathrm{Na}$-ion, which leads to higher ionic conductivity and greater mobility in the electrolyte ${ }^{10}$. Meanwhile, the formation enthalpy of $\mathrm{KC}_{8}\left(-27.5 \mathrm{~kJ} \mathrm{~mol}^{-1}\right)$ is lower than that of $\mathrm{LiC}_{6}\left(-16.5 \mathrm{~kJ} \mathrm{~mol}^{-1}\right)^{11}$, which means that $\mathrm{K}$ ions are easier to insert into graphite. Above all, the $\mathrm{K}$-ion transportation in the electrolyte is a kinetic-favorable process. However, the diffusion rate of $\mathrm{K}$-ion in the interior of the electrode is still significantly restricted by its large ionic radius ( $1.38 \AA$ vs. $1.02 \AA$ of Na-ion and $0.76 \AA$ of Li-ion), which will induce severe volume expansion and structure collapse of the electrode material during repeat potassiation/de-potassiation processes ${ }^{12}$. For example, the formation of $\mathrm{KC}_{8}$ compound endures a $\sim 61 \%$ volume expansion, which is six times of the lithiated graphite $\left(\mathrm{LiC}_{6}\right)^{13}$. To overcome this obstacle, adjusting the interlayer distance and decreasing the $\mathrm{K}$-ion diffusion distances of carbons could effectively ameliorate the volume expansion and avoid the structural collapse $\mathrm{e}^{2,14,15}$.

In addition to PIBs with promising prospect. So far, many kinds of rechargeable $\mathrm{Zn}$-ion energy storage devices, such as $\mathrm{Zn}$-ion capacitors, $\mathrm{Zn}$-ion batteries and $\mathrm{Zn}$-air batteries, have been widely studied owing to their high safety based on the aqueous electrolyte ${ }^{16-18}$. Among them, aqueous $\mathrm{Zn}$ ion hybrid supercapacitor (ZHS) is a newly-emerged energy storage device, which is usually composed of zinc metal anode and carbon-based cathode. ZHS is considered as one of the most attractive energy storage devices by combining the merits of batteries and supercapacitors ${ }^{19-22}$. For instance, Zhang et al. ${ }^{21}$ studied the use of hierarchically porous carbon as cathode in ZHS, which displayed a high energy density of $107.3 \mathrm{Wh} \mathrm{kg}^{-1}$ and a superb power density of $24.9 \mathrm{~kW} \mathrm{~kg}^{-1}$.

Herein, calcium gluconate was firstly introduced as a carbon source for constructing ultrathin hierarchically porous carbon nanosheets with a facile in situ template method. The specific surface 
area and porosity of carbon materials can be precisely tuned by adjusting the calcination temperatures. The as-obtained Ca-900 electrode exhibits superior K-ion storage performance including high reversible capacity (497.7 $\left.\mathrm{mAh} \mathrm{g}^{-1}\right)$, high rate capability $\left(5.0 \mathrm{~A} \mathrm{~g}^{-1}\right)$ and ultra-long cycling stability (4000 cycles at $1.0 \mathrm{~A} \mathrm{~g} \mathrm{~g}^{-1}$ ). The superiority and universality of the Ca-900 electrode is further verified in zinc-ion hybrid supercapacitor, which delivers high energy density (134.5 Wh $\left.\mathrm{kg}^{-1}\right)$ and power density $\left(9.3 \mathrm{~kW} \mathrm{~kg}^{-1}\right)$ as well as a high capacitance retention ( $\left.\sim 90.9 \%\right)$ over 4000 cycles. Moreover, to excavate the origin of the excellent electrochemical performance, a carefully designed consecutive CV measurement was introduced. The predominant capacitive behaviors, rooting in the high specific surface area and highly developed porous structure, are confirmed the main contribution of the unprecedent electrochemical performance. Overall, this work may open a new window for understanding the metal-ion storage behaviors with carbonaceous electrode materials. 


\section{Experimental Section}

2.1 Preparation of Ca-x materials: Ultrathin porous carbon nanosheets are prepared by using calcium gluconate $\left(\mathrm{Ca}\left(\mathrm{C}_{6} \mathrm{H}_{11} \mathrm{O}_{7}\right)_{2}\right)$ as the carbon source followed by a carbonization treatment and an acid etching process. Specifically, a certain amount of calcium gluconate powder $(5.0 \mathrm{~g})$ is weighed and placed evenly in a quartz boat, which is subsequently transferred to the tube furnace and pyrolyzed at selected temperatures $\left(600,700,800,900\right.$ and $1000{ }^{\circ} \mathrm{C}$, holding for $\left.1 \mathrm{~h}\right)$ with a temperature ramp rate of $5{ }^{\circ} \mathrm{C} \mathrm{min}^{-1}$ under Ar flow protection $\left(100 \mathrm{~mL} \mathrm{~min}^{-1}\right)$. After natural cooling, the precursors can be obtained (denoted as Ca-x-P, x stands for the carbonization temperature). Afterwards, the precursors are washed for three times with diluted hydrochloric acid $(\sim 1.2 \mathrm{~mol} / \mathrm{L})$, deionized water and anhydrous ethanol, respectively. After a vacuum drying process at $70{ }^{\circ} \mathrm{C}$ for $10 \mathrm{~h}$ followed by a soft grinding process in the mortar, the ultrathin porous carbon materials are collected and denoted as Ca-x.

2.2 Structural characterization: X-Ray Diffraction (XRD) analysis was performed on a Rigaku Miniflex 600 X-ray diffractometer with $\mathrm{Cu} \mathrm{K \alpha}$ radiation $(\lambda=1.5406 \AA)$ at a scan rate of $5 \% \mathrm{~min}$. Morphological and microstructural characterizations were conducted on Scanning electronic microscope (SEM, Phenom Pro and JEOL JSM-7610F) and Transmission electronic microscope (TEM, JEOL JEM-2100). The specific surface area and pore structure information were collected by $\mathrm{N}_{2}$ adsorption isotherm at $-196{ }^{\circ} \mathrm{C}$ on a Micromeritics ASAP 2020 analyzer. The samples were dried and degassed at $200{ }^{\circ} \mathrm{C}$ for 8 hours before testing. Raman spectra were collected on a Rennishaw inVia confocal Raman spectrometer using a $633 \mathrm{~nm}$ laser as the excitation source.

\subsection{Electrochemical measurement}

Potassium-ion batteries: Electrode materials are prepared by mixing $80 \mathrm{wt} \%$ active material (asprepared Ca-x), $10 \mathrm{wt} \%$ super $\mathrm{P}$ and $10 \mathrm{wt} \%$ polyvinylidene fluoride (PVDF) in the NMP (Nmethyl-2-pyrrolidone) solvent under magnetic stirring to form a uniform slurry. Afterward, the slurry is coated onto a copper foil followed by a vacuum drying process $\left(100{ }^{\circ} \mathrm{C}\right.$ for $\left.10 \mathrm{~h}\right)$ to evaporate the solvent. After natural cooling, the dried foil is cut into circular electrodes with a diameter of $12 \mathrm{~mm}$. K-ion cell is assembled in an argon-filled glovebox using CR2032-type coin cells with K-metal foil as the counter electrode. A whatman GF/D glass fiber filter is applied as the separator. The electrolyte is $0.8 \mathrm{M} \mathrm{KPF}_{6}$ dissolved in ethylene carbonate and diethyl carbonate ( $\mathrm{EC} / \mathrm{DEC}=1: 1$ by volume). The electrochemical measurements are evaluated within a voltage range of $0.01 \sim 3.0 \mathrm{~V}$ using a LAND-CT2001 A multichannel galvanostat (Wuhan, China) at $25^{\circ} \mathrm{C}$. Cyclic voltammetry $(\mathrm{CV})$ is acquired on a $\mathrm{CHI} 660 \mathrm{E}$ electrochemical workstation.

Zinc-ion capacitors: The electrode is prepared by mixing active material, conductive material carbon (super P) and polytetrafluoroethylene (PTFE) in the mass proportion of 85:10:5 with NMP as solvent followed by magnetic stirring to form a homogeneous slurry. The slurry is evenly smeared on the cleaned carbon paper followed by a vacuum drying process at $100^{\circ} \mathrm{C}$ for $10 \mathrm{~h}$. After natural 
cooling, the dried carbon paper is cut into circular electrodes with a diameter of $12 \mathrm{~mm}$. Zinc foil is applied as the counter electrode, commercial filter paper is used as the separator, and the electrolyte is $1.0 \mathrm{M}$ zinc sulfate $\left(\mathrm{ZnSO}_{4}\right)$ dissolved in deionized water. All capacitors are assembled with CR2032-type coin cells. The electrochemical performance is evaluated on LAND-CT2001A multichannel galvanostat within the voltage range of $0 \sim 1.8 \mathrm{~V}$, and the $\mathrm{CV}$ test results are obtained by a CHI660E electrochemical workstation. 


\section{Results and discussion}

\subsection{Morphological and microstructural analyses}

The synthetic process of the ultra-thin carbon nanosheets is illustrated in Fig. 1. Calcium gluconate was introduced as the carbon source. By controlling the calcination temperatures, various kinds of Ca-based hard template can be in situ generated. Followed by an acid etching process, the template can be easily removed and porous carbon nanosheets can be obtained. TEM observations were conducted to reveal the morphological evolutions. As shown in Fig. 1 and Fig. S1, after the calcination treatment, evenly distributed nanoparticles can be clearly distinguished on the ultrathin carbon nanosheets, which vanish after the acid etching process and ultrathin carbon nanosheets with uniformly distributed nanosized pores can be obtained.



Fig.1 Schematic illustrations of the synthetic process. (a) SEM images of calcium gluconate. (b) TEM image of Ca900-P and (c) Ca-900.

The TEM images of Ca-x exhibit a translucent characteristic (Fig. 1c and S1), indicating an ultrathin feature. The SEM images further verify this morphology (Fig. 2a-b and S2), the obtained Ca-x at various temperatures all exhibit an ultrathin thickness in the range of 20 30 nm (Fig. 2c), which will significantly shorten the diffusion lengths of tested metal ions and indicate a fast transport kinetics. XRD was further employed to track the phase transformations. As depicted in Fig. 2d, the in situ generated template can be determined as $\mathrm{CaCO}_{3}(\mathrm{PDF} 47-1743)$ under pyrolyzed temperatures below $800^{\circ} \mathrm{C}$, which further decomposes into $\mathrm{CaO}(\mathrm{PDF} \# 48-1467)$ and $\mathrm{CO}_{2}$ at higher temperatures. The detected $\mathrm{Ca}(\mathrm{OH})_{2}$ (PDF\#44-1481) signals at high temperatures $\left(800-1000^{\circ} \mathrm{C}\right)$ should be ascribed to the moisture sensitive feature of $\mathrm{CaO}$. Except the template virtue of the $\mathrm{Ca}$ based nanoparticles, the released $\mathrm{CO}_{2}$ gas can also play a vital role in creating micropores by etching the carbon matrix, which will be verified and discussed in Fig. 3. The involved reactions are summarized as follows:

$$
\begin{gathered}
\mathrm{CaCO}_{3} \rightarrow \mathrm{CaO}+\mathrm{CO}_{2} \\
\mathrm{CO}_{2}+\mathrm{C} \rightarrow \mathrm{CO}
\end{gathered}
$$


The EDS result is displayed in Fig. S3, the increasing C/O ratio (Ca-600 is 6.11 and Ca-900 is 9.91) at higher temperature indicates the decomposition of oxygen-containing groups, which will benefit the electronic conductivity. At this point, $\mathrm{Ca}$-x obtained at relatively high temperature will exhibit better battery performance. XRD patterns after the acid etching process were displayed in Fig. 2e. The obtained Ca-x all exhibit a broad peak at $\sim 22.8^{\circ}$ and a weak peak at $\sim 44^{\circ}$, corresponding to the (002) and (101) crystal indexes of graphite. The broad peak indicates a relatively amorphous structure of the porous carbon nanosheets. Besides, the lower angel shifted (002) peak compared with the standard pattern (PDF\#75-1621) means an enlarged interlayer spacing, which can be determined by the Bragg equation ${ }^{23}$ :

$$
2 d \sin \theta=\lambda
$$

And the interlayer spacing can be obtained by the deformation:

$$
d=\frac{\lambda}{2 \sin \theta}
$$

Herein, $\lambda$ is the wave length of the $\mathrm{Cu} \mathrm{K} \alpha$ radiation $(=1.5406 \AA)$. Therefore, the interlayer spacing of the obtained Ca-x is calculated to be $\sim 0.39 \mathrm{~nm}$, higher than graphite $(\sim 0.34 \mathrm{~nm})$. The expanded lamellar spacing is conducive to the rapid diffusion of metal ions, especially for $\mathrm{K}$ ions with larger ionic radius. Hence an enhanced rate capability can be expected.
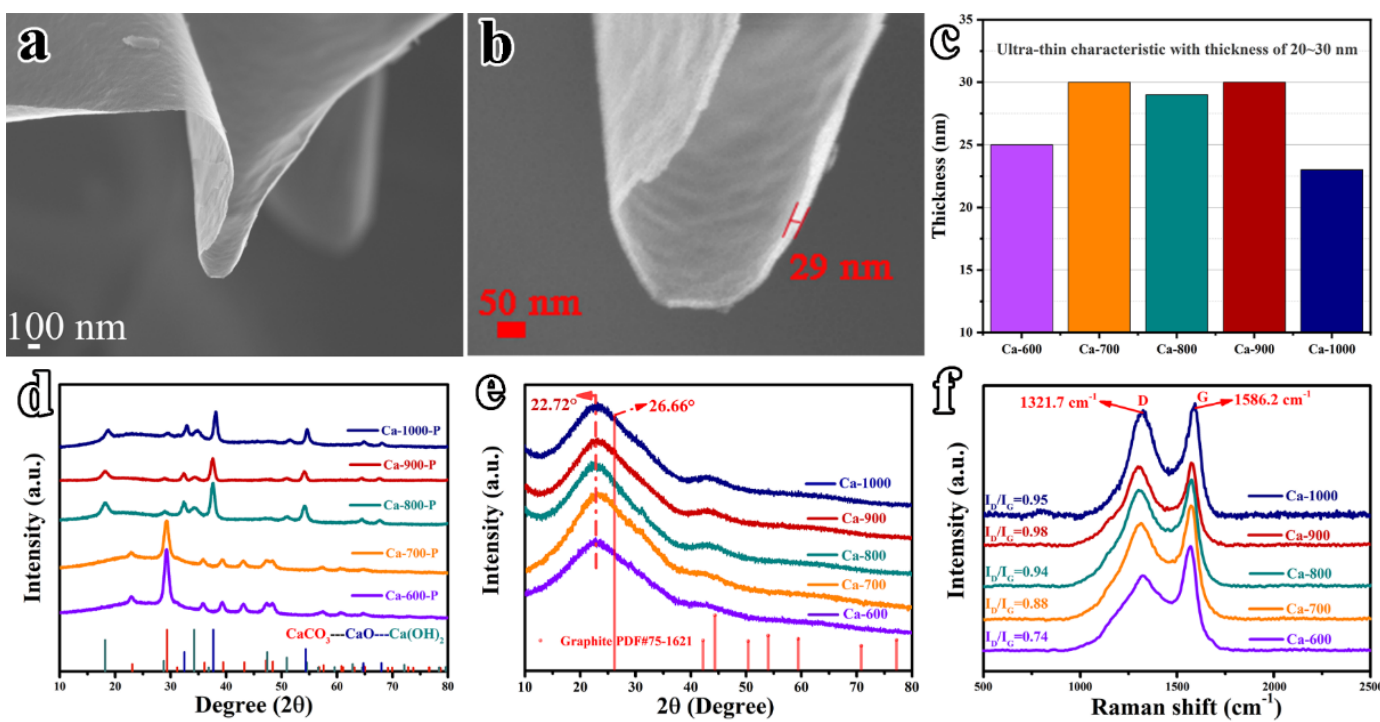

Fig.2 Morphological and structural characterizations. (a-b) SEM images of Ca-900. (c) A survey of the thickness of Ca-X. XRD patterns of (d) precursors Ca-X-P and (e) obtained carbon nanosheets Ca-X. (f) Raman spectra of Ca-X.

Moreover, Raman spectra were collected to grasp a better understanding of the carbon structure. As shown in Fig. 2f, two obvious peaks near $1322 \mathrm{~cm}^{-1}$ and $1586 \mathrm{~cm}^{-1}$ can be observed, corresponding to the characteristic D band and $\mathrm{G}$ band of graphite. D band represents the defect or disorder degree, while $\mathrm{G}$ band reflects the in-plane stretching vibration of the $\mathrm{sp}^{2}$ hybrid structure. Therefore, the $\mathrm{I}_{\mathrm{D}} / \mathrm{I}_{\mathrm{G}}$ ratio can interpret the degree of graphitization or disorder. Specifically, the calculated ratios increase with higher carbonization temperature (from 0.74 of Ca-600 to 0.95 of 
Ca-1000), indicating a more disorder/defective structure, which will provide sufficient accessible sites and diffusion pathways for the fast diffusion of metal ions.
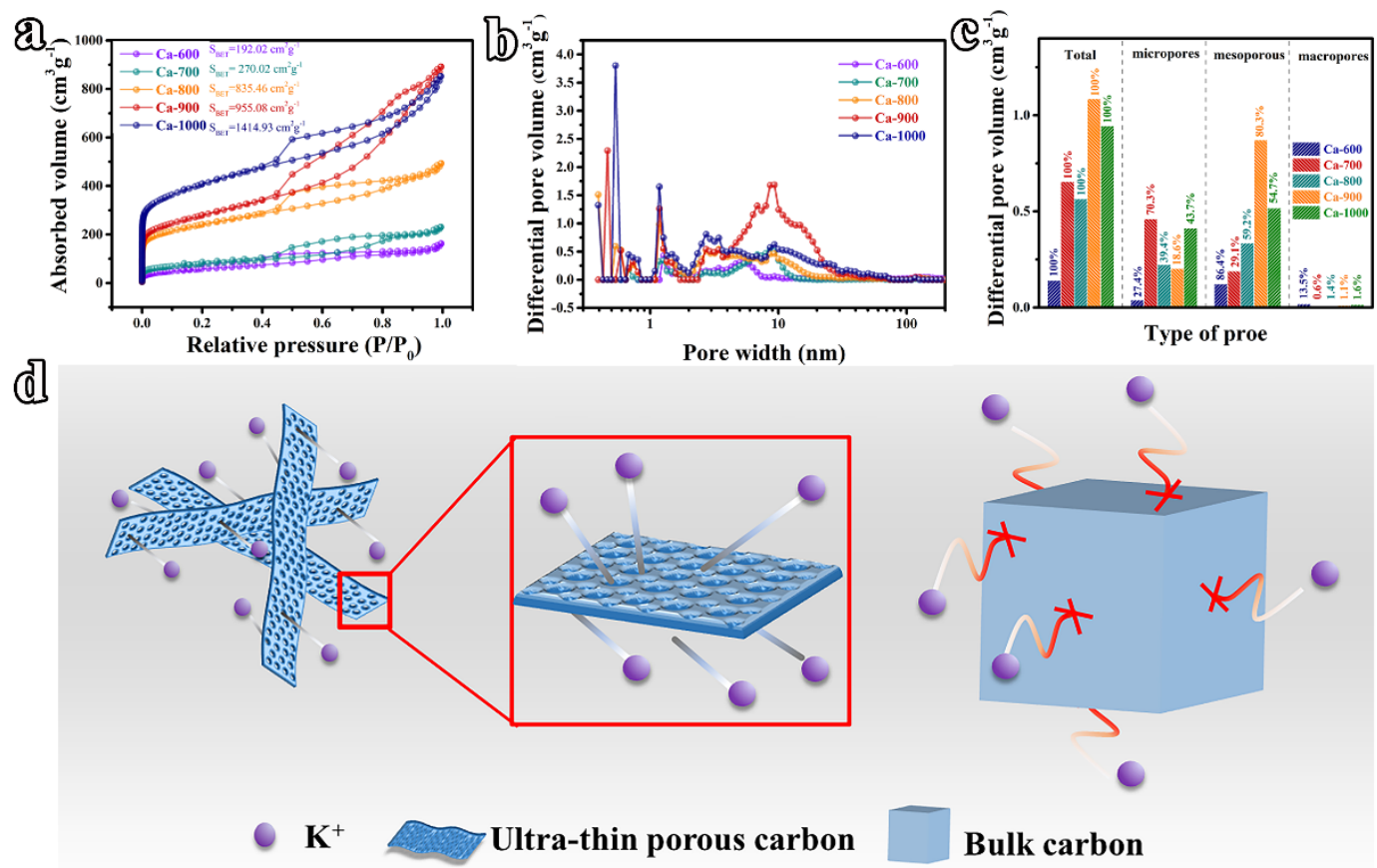

Fig.3 Pore structure evaluations. (a) Nitrogen adsorption-desorption isotherms. (b) Pore size distributions. (c) Pore volume contribution ratios of micropores, mesopores and macropores. (d) Schematic diagram of K-ion diffusion kinetics in the hierarchically porous ultrathin carbon nanosheets.

In the case of carbonaceous materials, a high disorder degree is always associated with a well developed porous structure ${ }^{24}$. As plotted in Fig 3a. the $\mathrm{N}_{2}$ adsorption-desportion curves of Ca-x all exhibit a combination of type I (uptake at the low $\mathrm{P} / \mathrm{P}_{0}$ region, characteristic of micropores) and type IV (hysterisis loop at the low $\mathrm{P} / \mathrm{P}_{0}$ region, characteristic of mesopores) isotherms. Besides, the uplift of the curve at high $\mathrm{P} / \mathrm{P}_{0}$ region corresponds to the existencce of macropores and this morphology has been clearly distinguished by SEM and TEM results (Fig. 1, Fig. S1 and Fig. 2a-b). Therefore, all of the Ca-x samples exhibit hierarchically porous structures with high specific surface areas (192.02, 270.02, 835.46, 955.08 and $1414.93 \mathrm{~m}^{2} \mathrm{~g}^{-1}$ for Ca-600, Ca-700, Ca-800, Ca-900 and Ca1000 , respectively). Among them, the hysteresis loop area of Ca-900 is the largest, which indicates abundant existence of mesopores. This structure provides sufficient channels and spaces for the diffusion of metal ions and alleviation of the volume expansion, which further contributes to enhanced rate performance and cycling stability 25,26 .

The unique porous structure is further dissected by the results of pore size distributions. As plotted in Fig. 3b, the hierarchically porous structure is solidified with peaks centered at the whole region of $0.4 \sim 100 \mathrm{~nm}$. Specifically, Ca-900 shows the strongest peak centered at $\sim 10 \mathrm{~nm}$, which is in accordance with the results of Fig. 3a. Besides, Ca-x samples obtained at high temperatures ( $800^{\circ} \mathrm{C}$ ) exhibit stronger peaks centered at micropore and mesopore region, which should originate from the decomposition process of $\mathrm{CaCO}_{3}$ at high temperatures. The in situ generated $\mathrm{CO}_{2}$ gas is a 
very effective pore-forming agent (similar as $\mathrm{KOH}$ ) by etching the carbon matrix (see formula 2), which is conducive to create pores, especially micropores. Moreover, the higher the temperature, the higher the pore-making activity of $\mathrm{CO}_{2}$, which is consistent with the results of Raman spectra. Abundant micropores serve as defective sites and express a higher $I_{D} / I_{G}$ value. Furthermore, the pore volume of Ca-x is concluded in Fig. 3c, consistently, Ca-900 exhibits the highest pore volume $\left(1.08 \mathrm{~cm}^{3} \mathrm{~g}^{-1}\right)$ and highest proportion of mesopores (80.3\%), indentifying its highly developed porous structure.

Briefly speaking, the facilely prepared $\mathrm{Ca}-\mathrm{x}$ samples with ultrathin hierarchically porous structure exhibit great potentials for large-sized K-ion storage (Fig. 3d), the reasons can be concluded as follows: 1) High specific surface areas (SSA) can guarantee sufficient contact between the electrolyte and electrode surface; 2) Highly developed porous structure can provide sufficient and broad K-ion diffusion channels, thus enabling fast and stable K-ion diffusion. 3) Abundant micropores alongwith high SSA can serve as extra sites for capacitive K-ion adsorption, improving the reversible specifica capacities. 4) Ultrathin characteristic shortens the diffusion lengths and ensures fast $\mathrm{K}$-ion transport kinetics.

\subsection{Electrochemical performance}

The cycling performance of Ca-x based PIBs was firstly evaluated (Fig. 4a). After being cycled at $100 \mathrm{~mA} \mathrm{~g}^{-1}$ for 100 cycles, Ca-900 electrode delivers the highest capacity of $234.3 \mathrm{mAh} \mathrm{g}^{-1}$, while the rest electrodes can only maintain capacities lower than $200 \mathrm{mAh} \mathrm{g}^{-1}$. Simultaneously, it can be observed that the coulombic efficiencies of all samples in the initial cycles are relatively low and gradually increase to $100 \%$ in the following cycles. This phenomenon can be attributed to the large SSA which consumes more electrolyte to form a stable SEI (solid electrolyte interface) layer on the electrode surface. In view of the unique ultrathin and porous structure of $\mathrm{Ca}-\mathrm{x}$, the rate capability was then investigated. As depicted in Fig. 4b, Ca-900 electrode delivers higher capacities than the rest electrodes at all current densities. The specific values of reversible capacities at each current density are concluded in Fig. 4c. Particularly, Ca-900 electrode can release a high capacity of 497.7 $\mathrm{mAh} \mathrm{g}^{-1}$, while the rest electrodes can only deliver capacities below $400 \mathrm{mAh} \mathrm{g}^{-1}$. Besides, Ca-900 electrode can suffer fast potassiation/de-potassiation shock and maintain a relatively high capacity of $154.8 \mathrm{mAh} \mathrm{g}^{-1}$ at ultrahigh current density of $5.0 \mathrm{~A} \mathrm{~g}^{-1}$, corresponding to the highest capacity retention ratio of $31.1 \%$ among all the $\mathrm{Ca}-\mathrm{x}$ electrodes. When the current density is set back to $0.05 \mathrm{~A} \mathrm{~g}^{-1}$, the reversible capacity of Ca-900 electrode can also recover to $450.8 \mathrm{mAh} \mathrm{g}^{-1}(84.4 \%$ retention), further verifying the superior electrode stability of Ca-900 at high current densities, while the rest electrodes suffer sever structural deformation and gradually lose the electrochemical activity.

Intrigued by the excellent rate capability of Ca-900 electrode, the long-term cycling stability was evaluated at high current density of $1.0 \mathrm{~A} \mathrm{~g}^{-1}$. As shown in Fig. 4d, Ca-900 electrode delivers a capacity retention ratio of $72.4 \%$ after 4000 cycles, corresponding to a capacity loss ratio of $0.034 \%$ 
per cycle, outmatching most of the reported carbon electrodes ${ }^{27}$.
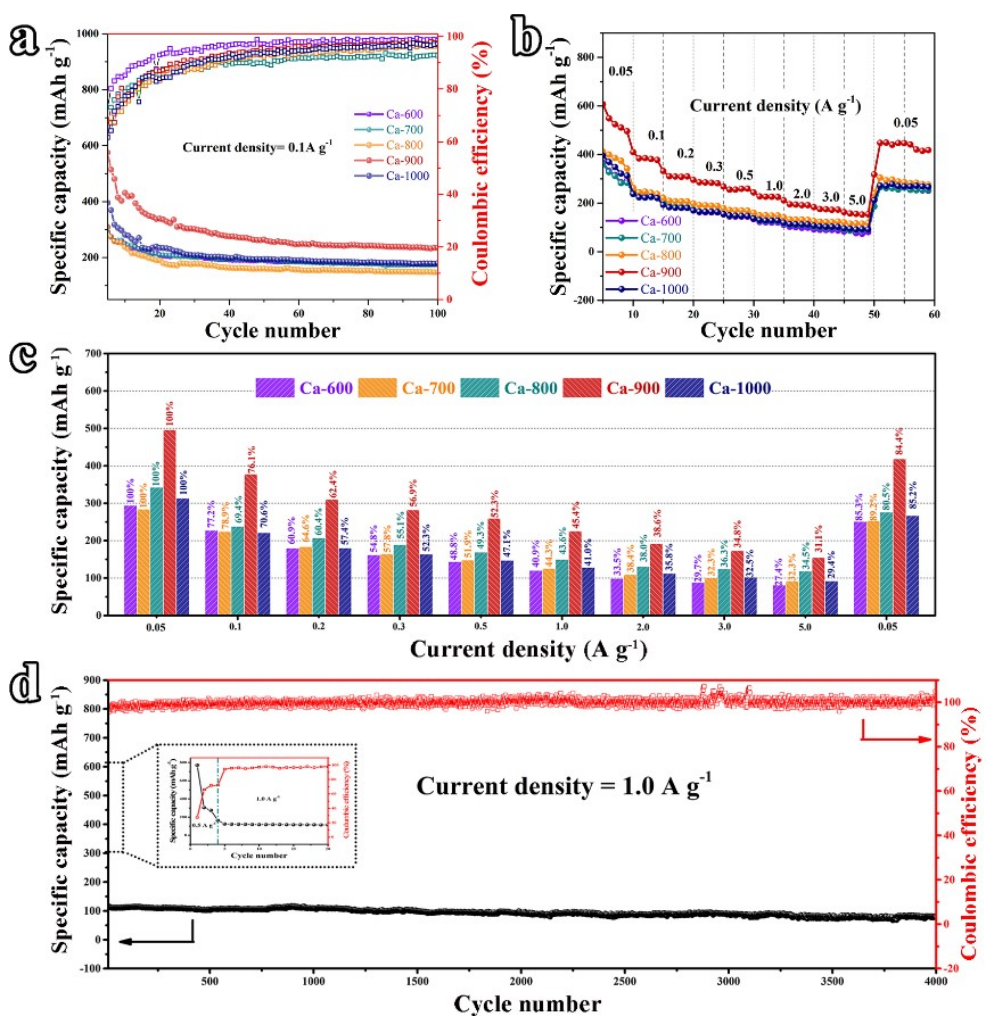

Fig.4 Electrochemical performance of Ca-x electrodes in PIBs. (a) Cycling performance. (b) Rate capability at various current densities. (c) Capacity retention ratios at various current densities. (d) Long-term cycling stability at $1.0 \mathrm{~A} \mathrm{~g}^{-1}$ (the electrode was preactivated at $0.05 \mathrm{~A} \mathrm{~g}^{-1}$ for 3 cycles).

To track the origin of the superior rate capability, consecutive CV measurements were conducted at various scan rates of 0.1 to $1.0 \mathrm{mV} \mathrm{s}^{-1}$ (Fig. 5a and Fig. S4). The corresponding relationship between peak current and scan rate can be obtained by these curves: $\boldsymbol{i}=\boldsymbol{a} \boldsymbol{v}^{\boldsymbol{b}}$, where $\boldsymbol{a}$ and $\boldsymbol{b}$ are adjustable constants. The $\boldsymbol{b}$ value can be obtained from the slope by plotting $\log (\boldsymbol{i}) \boldsymbol{v s} \cdot \log$ $(v)^{28,29}$. Specifically, when $\boldsymbol{b}$ value is approaching 0.5 , the tested electrode is dominated by the battery behavior (relatively sluggish), corresponding to the diffusion process. When $\boldsymbol{b}$ value is close to 1.0, capacitive behavior predominates alongwith the adsorption process (kinetic favorable) ${ }^{30}$. The calculated $\boldsymbol{b}$ values are concluded in Fig. 5b and Fig. S4, it can be observed that $\boldsymbol{b}$ value varies at different potassiation depths. To parse this phenomenon, the calculated $\boldsymbol{b}$ values at various potentials are concluded in Fig. 5c, and we come to a preliminary conjecture that capacitive K-ion storage process mainly takes place within the potential range of $0.4 \sim 2.0 \mathrm{~V}\left(\mathrm{vs} . \mathrm{K} / \mathrm{K}^{+}\right)$with higher $\boldsymbol{b}$ values. Moreover, the mixed behaviors were quantified by the following equations: $\boldsymbol{i}=\boldsymbol{k}_{\mathbf{1}} \boldsymbol{v}+\boldsymbol{k}_{\mathbf{2}} \sqrt{\boldsymbol{v}}$. Herein, $\boldsymbol{k}_{1}$ and $\boldsymbol{k}_{2}$ are constants, $\boldsymbol{k}_{\mathbf{1}} \boldsymbol{v}$ stands for the capacitive contribution, while $\boldsymbol{k}_{2} \sqrt{\boldsymbol{v}}$ represents the contribution of diffusion-controlled behavior. As plotted in Fig. 5d and Fig. S4, the red part represents the capacitive contribution, which dominates the total capacity $(66.02 \%$ for Ca-900, higher than the rest electrodes). Specially, the red part occupies a higher ratio in the region higher than $0.4 \mathrm{~V}$, consistent with the variation tendency of $\boldsymbol{b}$ values. Moreover, the capacitive contribution 
ratios of all Ca-x samples are concluded in Fig. 5e, Ca-900 electrode is the highest at all scan rates, which is also consistent with its best electrochemical performance.
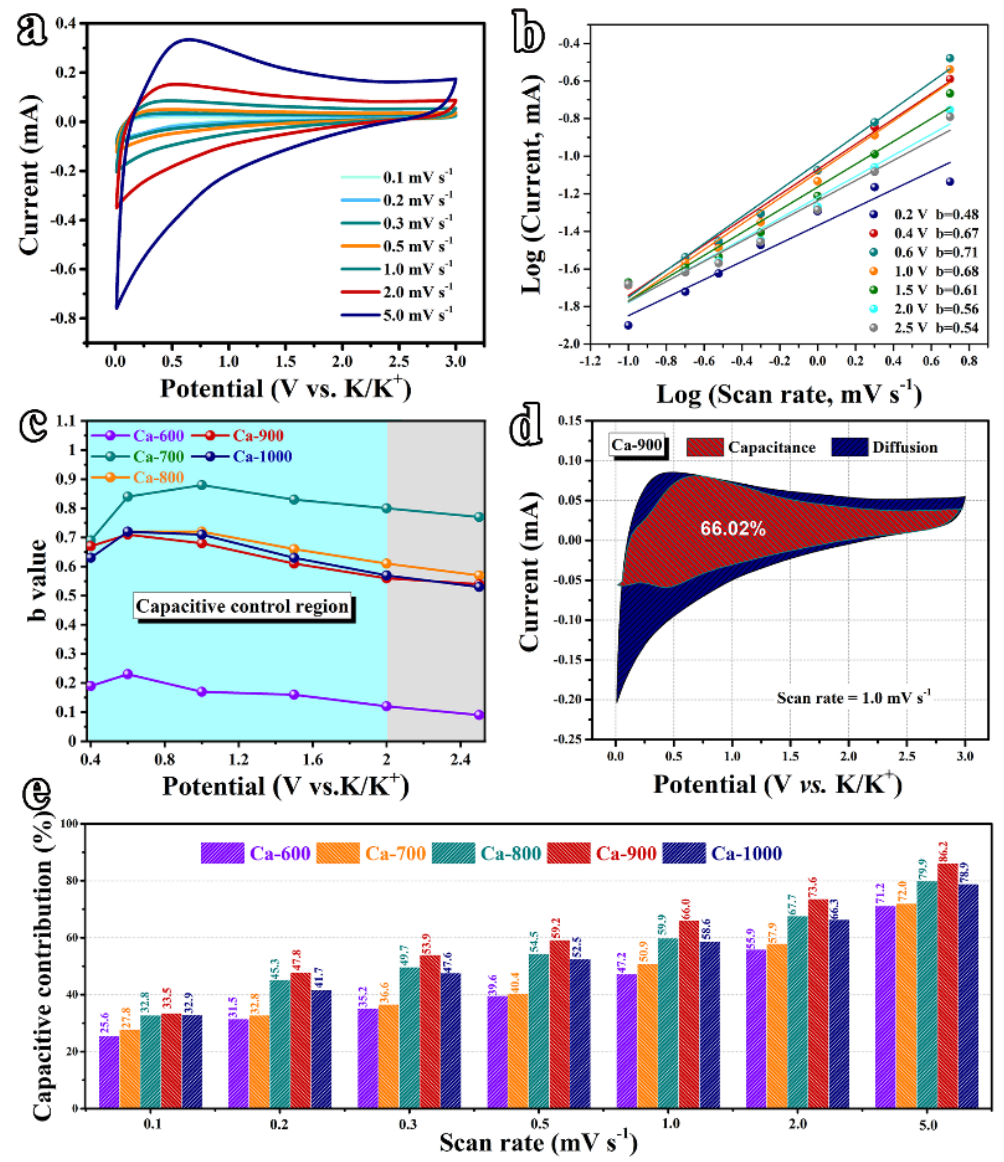

Fig.5 Kinetic evaluations of Ca-x electrodes in PIBs. (a) CV curves of Ca-900 electrode at various scan rates. (b) $b$ values of Ca-900 electrode determined at various potassiation depths. (c) b values of all Ca-x electrodes. (d) Sketch of the capacitive contribution at $1.0 \mathrm{mV} \mathrm{s}^{-1}$. (e) Capacitive contribution ratios of $\mathrm{Ca}-\mathrm{x}$ electrodes at various scan rates.

Zinc-ion hybrid supercapacitors (ZHSs) have received extensive attentions owing to the rich zinc sources, low cost, and high safety ${ }^{22,31}$. The negative electrode material for ZHSs is usually a carbon-based material with a high specific surface area $^{32,33}$. Therefore, our carefully designed Ca900 with unique ultrathin hierarchically porous structure shows great potential in ZHSs.

$\mathrm{CV}$ measurements were firstly conducted at different scan rates $\left(5.0 \mathrm{mV} \mathrm{s}^{-1} \sim 200 \mathrm{mV} \mathrm{s}^{-1}\right)$ to investigate the Zn-ion storage behavior. As shown in Fig. 6a, weak redox peaks can be observed, representing the pseudo-capacitive behavior. Similarly, the $\boldsymbol{b}$ values can be extracted from the CV curves at various potentials (Fig. 6b), which are undoubtedly higher than that of PIBs, indicating the capacitive inherence of $\mathrm{ZHSs}^{34}$. The contribution of capacitive behavior was plotted in Fig. 6c, unlike the plot of PIBs, the red part distributed evenly within the tested potential range, further confirming the capacitive nature of ZHSs. Besides, Ca-900 electrode delivers high capacitive contribution ratios at all scan rates, especially at a superhigh scan rate of $200 \mathrm{mV} \mathrm{s}^{-1}$ (Fig. 6d). Consequently, Ca-900 electrode exhibits a good rate capability with a high capacity retention ratio of $\sim 40 \%$ at $2.0 \mathrm{~A} \mathrm{~g}^{-1}$. (Fig. 6e and Fig. S5). Besides, when current density returns to $0.1 \mathrm{~A} \mathrm{~g}^{-1}$, the $12 / 16$ 
capacity is just slightly lower than the initial cycles, indicating a high reversibility. To study the practical prospect, the energy density and power density were calculated and concluded as Ragone plot in Fig. 6f. When power density is $1.8 \mathrm{~kW} \mathrm{~kg}^{-1}$, a high energy density of $134.5 \mathrm{Wh} \mathrm{kg}^{-1}$ is achieved. When power density increases to $9.3 \mathrm{~kW} \mathrm{~kg}^{-1}$, the power density is still as high as 98.6 Wh $\mathrm{kg}^{-1}$. Compared with reported carbon-based ZHSs, such as $\mathrm{HNPC} / / \mathrm{Zn}^{35}, \mathrm{AC} / / \mathrm{AC}^{36}$, Graphene//Graphene ${ }^{37}, \mathrm{MPCs} / / \mathrm{Zn}^{38}, \mathrm{RuO}_{2} \cdot \mathrm{H}_{2} \mathrm{O} / / \mathrm{Zn}^{22}, \mathrm{MDC} / / \mathrm{Zn}^{39}$, a-MEGO$/ / \mathrm{Zn}^{40}, \mathrm{HPC} / / \mathrm{Zn}^{41}$ and $\mathrm{HSC} / / \mathrm{Zn}^{19}$, our $\mathrm{Ca}-900$ electrode shows the best overall electrochemical performance. Moreover, Ca-900 electrode also exhibits superior cycling stability at a high current density of $1.0 \mathrm{~A} \mathrm{~g}^{-1}$ with a neglectable capacity loss ratio of $0.028 \%$ per cycle, further solidifying the effectiveness of the ultrathin porous structure in capacitive energy storage systems.


Fig.6 Electrochemical performance of Ca-900//Zn in Zn-ion capacitors. (a) CV curves at various scan rates. (b) $b$ values at various scan rates. (c) Sketch of capacitive contribution at $10 \mathrm{mV} \mathrm{s}^{-1}$. (d) Capacitive contribution ratios at various scan rates. (e) Rate performance at various current densities. (f) Ragone plots. (g) Long-term cycling performance.

\section{Conclusion}

In summary, we propose a facile preparation strategy for constructing ultrathin porous carbon materials by direct pyrolyzing calcium gluconate. The obtained carbons show ultrathin characteristic (thickness of 20 30 nm) and hierarchically porous structure (high SSA of $955.08 \mathrm{~m}^{2}$ $\mathrm{g}^{-1}$ and pore volume of $\left.1.08 \mathrm{~cm}^{3} \mathrm{~g}^{-1}\right)$. Therefore, the as-prepared Ca-900 electrode delivers excellent electrochemical performance in PIBs with high reversible capacity $\left(497.7 \mathrm{mAh} \mathrm{g}^{-1}\right)$, excellent rate capability (158.4 $\mathrm{mAh} \mathrm{g}^{-1}$ at $5.0 \mathrm{~A} \mathrm{~g}^{-1}$ ) and ultra-stable cycling performance (over 4000 cycles at 1.0 
$\mathrm{A} \mathrm{g}^{-1}$ ). In virtue of the carefully designed consecutive $\mathrm{CV}$ measurements, it is confirmed that the unique porous structure contributes to predominant capacitive metal-ion storage, thus resulting in the significantly improved battery performance. Moreover, we dissect the mixed behaviors at various potassiation depths and we draw a conclusion that capacitive behavior dominates in the range of $0.4 \sim 2.0 \mathrm{~V}$ with carbon-based anode materials. Furthermore, Ca-900 electrode also exhibits remarkable $\mathrm{Zn}$-ion storage capability with a capacity retention ratio of $\sim 90.9 \%$ after 4000 cycles at $1.0 \mathrm{~A} \mathrm{~g} \mathrm{~g}^{-1}$. Considering the facile preparation strategy, unique microstructure, outstanding electrochemical performance and in-depth mechanism investigations, we believe this work will strength the fundamental understandings of porous carbons and boost the development of carbonbased energy storage devices like K-ion/Na-ion/Li-ion batteries/capacitors, Al-ion batteries, Zn-ion capacitors and Dual-ion capacitors, etc.

\section{Acknowledgment}

This work was supported by High-level Talents' Discipline Construction Fund of Shandong University (Grant No. 31370089963078), School Research Startup Expenses of Harbin Institute of Technology (Shenzhen) (Grant No. DD29100027), China Postdoctoral Science Foundation (Grant No. 2019M661276), Guangdong Basic and Applied Basic Research Foundation (Grant No. 2019A1515110756).

\section{References}

1. Dunn, B.; Kamath, H.; Tarascon, J.-M., Electrical Energy Storage for the Grid: A Battery of Choices. Science 2011, 334 (6058), 928

2. Li, D.; Ren, X.; Ai, Q.; Sun, Q.; Zhu, L.; Liu, Y.; Liang, Z.; Peng, R.; Si, P.; Lou, J.; Feng, J.; Ci, L., Facile Fabrication of Nitrogen-Doped Porous Carbon as Superior Anode Material for Potassium-Ion Batteries. Advanced Energy Materials 2018, 8 (34), 1802386

3. Xu, Y.; Zhang, C.; Zhou, M.; Fu, Q.; Zhao, C.; Wu, M.; Lei, Y., Highly nitrogen doped carbon nanofibers with superior rate capability and cyclability for potassium ion batteries. Nature communications 2018, 9 (1), 1720.

4. Jian, Z.; Xing, Z.; Bommier, C.; Li, Z.; Ji, X., Hard carbon microspheres: potassium-ion anode versus sodiumion anode. Advanced Energy Materials 2016, 6 (3), 1501874.

5. Xu, Y.; Zhou, M.; Wang, X.; Wang, C.; Liang, L.; Grote, F.; Wu, M.; Mi, Y.; Lei, Y., Enhancement of Sodium Ion Battery Performance Enabled by Oxygen Vacancies. Angewandte Chemie International Edition 2015, 54 (30), $8768-8771$.

6. Wang, G.; Xiong, X.; Xie, D.; Lin, Z.; Zheng, J.; Zheng, F.; Li, Y.; Liu, Y.; Yang, C.; Liu, M., Chemically activated hollow carbon nanospheres as a high-performance anode material for potassium ion batteries. Journal of Materials Chemistry A 2018, 6 (47), 24317-24323.

7. Li, D.; Zhang, Y.; Sun, Q.; Zhang, S.; Wang, Z.; Liang, Z.; Si, P.; Ci, L., Hierarchically porous carbon supported $\mathrm{Sn}_{4} \mathrm{P}_{3}$ as a superior anode material for potassium-ion batteries. Energy Storage Materials 2019, 23, 367-374.

8. Wu, Y.; Huang, H.-B.; Feng, Y.; Wu, Z.-S.; Yu, Y., The Promise and Challenge of Phosphorus-Based Composites as Anode Materials for Potassium-Ion Batteries. Advanced Materials 2019, 31 (50), 1901414.

9. Zhang, W.; Mao, J.; Li, S.; Chen, Z.; Guo, Z., Phosphorus-Based Alloy Materials for Advanced Potassium-Ion Battery Anode. Journal of the American Chemical Society 2017, 139 (9), 3316-3319.

10. Tan, H.; Feng, Y.; Rui, X.; Yu, Y.; Huang, S., Metal Chalcogenides: Paving the Way for High-Performance 
Sodium/Potassium-Ion Batteries. Small Methods 2020, 4 (1), 1900563.

11. Wang, Z.; Selbach, S. M.; Grande, T., Van der Waals density functional study of the energetics of alkali metal intercalation in graphite. Rsc Advances 2014, 4 (8), 4069-4079.

12. Zhang, W.; Liu, Y.; Guo, Z., Approaching high-performance potassium-ion batteries via advanced design strategies and engineering. Science advances 2019, 5 (5), eaav7412.

13. Wang, L.; Guo, C.; Zhu, Y.; Zhou, J.; Fan, L.; Qian, Y., A FeCl2-graphite sandwich composite with $\mathrm{Cl}$ doping in graphite layers: a new anode material for high-performance Li-ion batteries. Nanoscale 2014, 6 (23), 14174-14179. 14. Wang, F.; Yi, J.; Wang, Y.; Wang, C.; Wang, J.; Xia, Y., Graphite Intercalation Compounds (GICs): A New Type of Promising Anode Material for Lithium-Ion Batteries. Advanced Energy Materials 2014, 4 (2), 1300600.

15. Wang, L.; Zhu, Y.; Guo, C.; Zhu, X.; Liang, J.; Qian, Y., Ferric chloride-Graphite Intercalation Compounds as Anode Materials for Li-ion Batteries. ChemSusChem 2014, 7 (1), 87-91.

16. Zhang, X.; Pei, Z.; Wang, C.; Yuan, Z.; Wei, L.; Pan, Y.; Mahmood, A.; Shao, Q.; Chen, Y., Flexible Zinc-Ion Hybrid Fiber Capacitors with Ultrahigh Energy Density and Long Cycling Life for Wearable Electronics. Small 2019, 15 (47), 1903817.

17. Xu, C.; Li, B.; Du, H.; Kang, F., Energetic Zinc Ion Chemistry: The Rechargeable Zinc Ion Battery. Angewandte Chemie 2012, 51 (4), 933-935.

18. Olsson, E.; Hussain, T.; Karton, A.; Cai, Q., The adsorption and migration behavior of divalent metals (Mg, $\mathrm{Ca}$, and $\mathrm{Zn}$ ) on pristine and defective graphene. Carbon 2020, 163, 276-287.

19. Wang, H.; Wang, M.; Tang, Y., A novel zinc-ion hybrid supercapacitor for long-life and low-cost energy storage applications. Energy Storage Materials 2018, 13, 1-7.

20. Dong, L.; Ma, X.; Li, Y.; Zhao, L.; Liu, W.; Cheng, J.; Xu, C.; Li, B.; Yang, Q.; Kang, F., Extremely safe, highrate and ultralong-life zinc-ion hybrid supercapacitors. Energy Storage Materials 2018, 13, 96-102.

21. Zhang, H.; Liu, Q.; Fang, Y.; Teng, C.; Liu, X.; Fang, P.; Tong, Y.; Lu, X., Boosting Zn-Ion Energy Storage Capability of Hierarchically Porous Carbon by Promoting Chemical Adsorption. Advanced Materials 2019, 31 (44), 1904948.

22. Dong, L.; Yang, W.; Yang, W.; Wang, C.; Li, Y.; Xu, C.; Wan, S.; He, F.; Kang, F.; Wang, G., High-Power and Ultralong-Life Aqueous Zinc-Ion Hybrid Capacitors Based on Pseudocapacitive Charge Storage. Nano-micro Letters 2019, 11 (1), 94.

23. Pope, C. G., X-Ray Diffraction and the Bragg Equation. Journal of Chemical Education 1997, 74 (1), 129 131.

24. Li, D.; Chen, L.; Chen, L.; Sun, Q.; Zhu, M.; Zhang, Y.; Liu, Y.; Liang, Z.; Si, P.; Lou, J., Potassium gluconatederived N/S Co-doped carbon nanosheets as superior electrode materials for supercapacitors and sodium-ion batteries. Journal of Power Sources 2019, 414, 308-316.

25. Li, D.; Sun, Q.; Zhang, Y.; Chen, L.; Wang, Z.; Liang, Z.; Si, P.; Ci, L., Surface-Confined SnS2@C@rGO as High-Performance Anode Materials for Sodium- and Potassium-Ion Batteries. ChemSusChem 2019, 12 (12), 26892700 .

26. Xiao, M.; Zhu, J.; Feng, L.; Liu, C.; Xing, W., Meso/Macroporous Nitrogen-Doped Carbon Architectures with Iron Carbide Encapsulated in Graphitic Layers as an Efficient and Robust Catalyst for the Oxygen Reduction Reaction in Both Acidic and Alkaline Solutions. Advanced Materials 2015, 27 (15), 2521-2527.

27. Wu, X.; Chen, Y.; Xing, Z.; Lam, C. W. K.; Pang, S.-S.; Zhang, W.; Ju, Z., Advanced Carbon-Based Anodes for Potassium-Ion Batteries. 2019, 9 (21), 1900343.

28. Cui, Y.; Liu, W.; Wang, X.; Li, J.; Zhang, Y.; Du, Y.; Liu, S.; Wang, H.; Feng, W.; Chen, M., Bioinspired Mineralization under Freezing Conditions: An Approach to Fabricate Porous Carbons with Complicated Architecture and Superior $\mathrm{K}^{+}$Storage Performance. ACS Nano 2019, 13 (10), 11582-11592. 
29. Liu, L.; Lin, Z.; Chane-Ching, J.-Y.; Shao, H.; Taberna, P.-L.; Simon, P., 3D rGO aerogel with superior electrochemical performance for K-Ion battery. Energy Storage Materials 2019, 19, 306-313.

30. Zhang, Y.; Chen, L.; Hao, C.; Zheng, X.; Guo, Y.; Chen, L.; Lai, K.; Zhang, Y.; Ci, L., Potassium pre-inserted $\mathrm{K}_{1.04} \mathrm{Mn}_{8} \mathrm{O}_{16}$ as cathode materials for aqueous Li-ion and Na-ion hybrid capacitors. Journal of Energy Chemistry 2020, $46,53-61$.

31. Wei, T.; Li, Q.; Yang, G.; Wang, C., Pseudo-Zn-Air and Zn-Ion Intercalation Dual Mechanisms to Realize High-Areal Capacitance and Long-Life Energy Storage in Aqueous Zn Battery. Advanced Energy Materials 2019, 9 (34), 1901480.

32. Zheng, Y.; Zhao, W.; Jia, D.; Liu, Y.; Cui, L.; Wei, D.; Zheng, R.; Liu, J., Porous carbon prepared via combustion and acid treatment as flexible zinc-ion capacitor electrode material. Chemical Engineering Journal 2020, $387,124161$.

33. Liu, P.; Gao, Y.; Tan, Y.; Liu, W.; Huang, Y.; Yan, J.; Liu, K., Rational design of nitrogen doped hierarchical porous carbon for optimized zinc-ion hybrid supercapacitors. Nano Research 2019, 12 (11), 2835-2841.

34. Chen, Y.; Qin, L.; Lei, Y.; Li, X.; Dong, J.; Zhai, D.; Li, B.; Kang, F., Correlation between Microstructure and Potassium Storage Behavior in Reduced Graphene Oxide Materials. ACS Applied Materials \& Interfaces 2019, 11 (49), 45578-45585.

35. Zhang, H.; Liu, Q.; Fang, Y.; Teng, C.; Liu, X.; Fang, P.; Tong, Y.; Lu, X., Boosting Zn-Ion Energy Storage Capability of Hierarchically Porous Carbon by Promoting Chemical Adsorption. Advanced Materials 2019, 31(44), 1904948.

36. Liu, J.; Guan, C.; Zhou, C.; Fan, Z.; Ke, Q.; Zhang, G.; Liu, C.; Wang, J., A flexible quasi-solid-state nickelzinc battery with high energy and power densities based on 3D electrode design. Advanced materials 2016, 28 (39), 8732-8739.

37. Dong, S.; Xu, Y.; Wu, L.; Dou, H.; Zhang, X., Surface-functionalized graphene-based quasi-solid-state Na-ion hybrid capacitors with excellent performance. Energy Storage Materials 2018, 11, 8-15.

38. Jiang, C.; Zou, Z., Waste polyurethane foam filler-derived mesoporous carbons as superior electrode materials for EDLCs and Zn-ion capacitors. Diamond and Related Materials 2019, 101, 107603.

39. Xiong, T.; Shen, Y.; Lee, W. S. V.; Xue, J., Metal Organic framework derived carbon for ultrahigh power and long cyclic life aqueous Zn ion capacitor. Nano Materials Science 2019, 2(2), 159-163.

40. Wu, S.; Chen, Y.; Jiao, T.; Zhou, J.; Cheng, J.; Liu, B.; Yang, S.; Zhang, K.; Zhang, W., An Aqueous Zn-Ion Hybrid Supercapacitor with High Energy Density and Ultrastability up to 80000 Cycles. Advanced Energy Materials 2019, 9(47), 1902915.

41. Yu, P.; Zeng, Y.; Zeng, Y.; Dong, H.; Hu, H.; Liu, Y.; Zheng, M.; Xiao, Y.; Lu, X.; Liang, Y., Achieving highenergy-density and ultra-stable zinc-ion hybrid supercapacitors by engineering hierarchical porous carbon architecture. Electrochimica Acta 2019, 327, 134999. 